\title{
HPV-18 E6*I protein modulates the E6-directed degradation of p53 by binding to full-length HPV-18 E6
}

\author{
David Pim*,1 and Lawrence Banks ${ }^{1}$ \\ ${ }^{1}$ International Centre for Genetic Engineering and Biotechnology, Area Science Park, Padriciano-99, I-34012 Trieste, Italy
}

We have previously demonstrated that ectopic expression of the HPV-18 E6*I protein has an antiproliferative effect in cells derived from HPV-containing cervical tumours. This effect correlated with the ability of E6*I to inhibit the E6-mediated degradation of p53 both in vitro and in vivo and with an increase in p53 transcriptional trans-activation. The observation that the E6*I protein can interact with both full-length HPV-18 E6 and E6-AP proteins in vitro indicated the mechanism by which this activity was mediated. In this study we describe a mutational strategy to attempt to differentiate between the E6-AP and full-length HPV-18 E6 interactions, with respect to the biological function of E6*I. We identify regions of the E6*I protein essential for its interaction with full-length E6 and important for its interaction with E6-AP. We show that a mutant of E6*I which is unable to bind to full-length HPV-18 E6 protein is unable to inhibit the E6-directed degradation of p53 and is also unable to inhibit the proliferation of a cervical tumour-derived cell line. Finally, we show that inhibition of transformed cell growth by E6*I protein correlates with its ability to induce apoptosis in a p53dependent manner. These results raise the intriguing possibility of using E6*I as a basis for therapeutic intervention in HPV-associated tumours.

Keywords: HPV E6; p53; E6*; apoptosis

\section{Introduction}

A subset of Human Papillomaviruses (HPVs) which infect the anogenital mucosa are found associated with lesions that can ultimately progress to the development of cervical carcinoma (zur Hausen and Schneider, 1987). The E7 proteins of these 'high risk' HPVs, by interacting with several key cell-cycle control proteins, including $\mathrm{pRB}$ (Dyson et al., 1989), induce the cell cycle re-entry of HPV-infected differentiating keratinocytes. The resulting unscheduled DNA replication is believed to lead to activation of p53-mediated cell-cycle arrest and apoptotic pathways. However, these HPVs overcome both p53-mediated responses by means of the interaction between their E6 proteins and p53, which ultimately leads to the ubiquitin-mediated degradation of p53 (Werness et al., 1990; Scheffner et al., 1990, 1993; Huibregtse et al., 1991). The reduced capacity of HPV-infected keratinocytes to mount a

*Correspondence: D Pim

Received 18 December 1998; revised 5 July 1999; accepted 6 August 1999 p53-mediated apoptotic response thus leaves them unable to respond effectively to subsequent genetic changes, and increases the risk of immortalization and progression to malignancy. However, despite the prevalence of infection by high risk HPV types, such as 16 and 18, only a small number of lesions progress to malignancy. This supports the notion of a balanced host-virus relationship, whereby the interaction between p53 and E6 may be modulated to minimize the risk of immortalization of the host cell, since this is usually associated with integration of the viral genome and loss of replicative competence.

The high risk, but not the low risk, HPVs transcribe their E6 and E7 genes as linear bicistronic and spliced polycistronic transcripts (Schwarz et al., 1985; Schneider-Gädicke and Schwarz, 1986; Smotkin and Wettstein, 1986). In infected keratinocytes and some celllines derived from HPV-containing cervical tumours, these spliced transcripts are more abundant than nonspliced, to the point that unspliced transcripts are difficult to detect (Doorbar et al., 1990; Böhm et al., 1993; Grassmann et al., 1996). These spliced transcripts originate from the viral early promoter and splice out at a splice donor site about $120 \mathrm{bp}$ from the initiation codon, then splice back in at, typically, four downstream splice acceptors to produce E6* I-IV mRNAs (Smotkin and Wettstein, 1986; Smotkin et al., 1989; Doorbar et al., 1990; Sherman and Alloul, 1992). This is shown schematically in Figure 1. For some time it was thought that this splicing event was a means of obtaining high levels of E7 expression, however recent evidence suggests that this is not the case (Stacey et al., 1995; Cheng et al., 1995), indicating that this splicing has a different, and more specific function.

We have previously shown that overexpression of HPV-18 E6*I in CaSKi cells has an antiproliferative effect, which was in marked contrast to the lack of effect in cells which were negative for p53 and/or E6 (Pim et al., 1997). We demonstrated that this antiproliferative activity of E6*I correlated with its ability to inhibit E6 mediated degradation of p53 both in vitro and in vivo and with the subsequent increase in p53 transcriptional transactivation. The observation that E6*I could bind to the full-length HPV-16 and HPV-18 E6 proteins and to E6-AP, but not to p53, appeared to explain the mechanism of E6*I activity. In the present study we have used a set of mutants of the HPV-18 E6*I protein in an attempt to identify which of these interactions might be essential for its biological properties. We show that one of these mutants, although reduced for E6-AP binding in vitro, is negative for binding to full-length HPV-18 E6. This same mutant is strongly reduced in its ability to inhibit the E6-directed degradation of p53 in vitro, and is also 

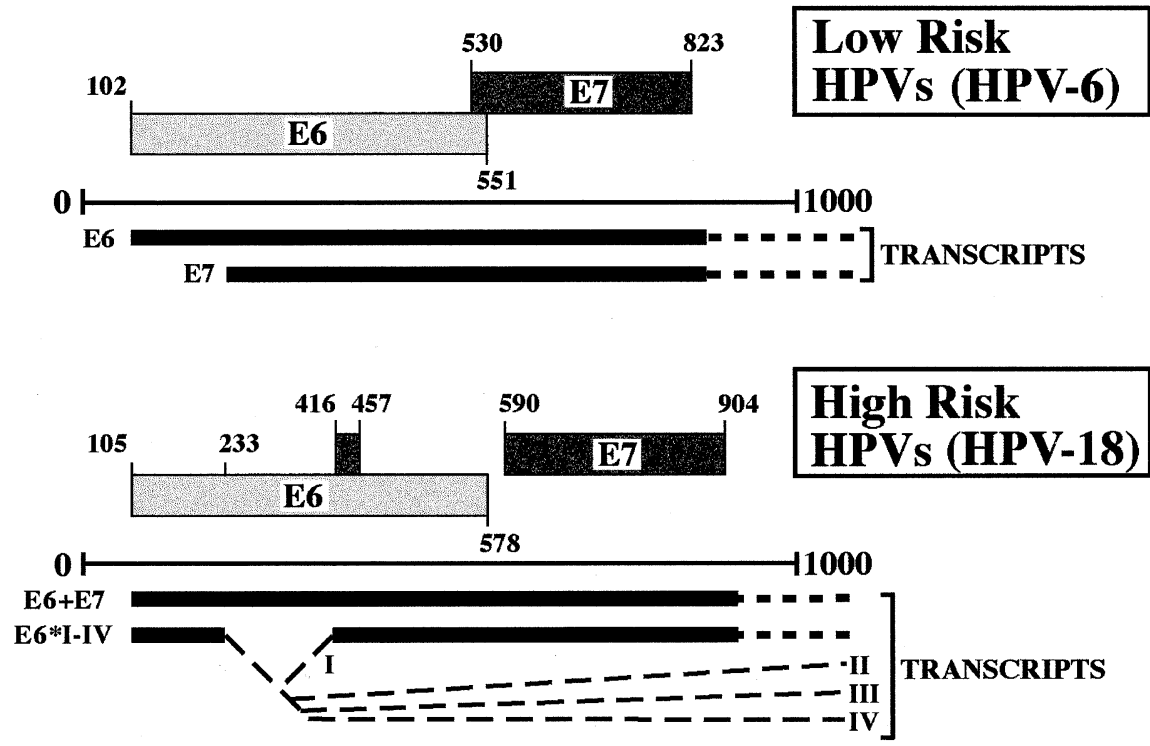

Figure 1 Schematic showing the origin of the different E6* mRNAs obtained from high risk HPVs compared with the transcription pattern obtained from low risk HPV types

defective in its ability to inhibit the proliferation of CaSKi cells. We also show that the inhibition of cell proliferation by wild-type E6*I correlates with its ability to induce apoptosis in a p53 dependent manner.

\section{Results}

Residues lying between amino acids 21 and 43 are required for HPV-18 E6*I binding to E6-AP and full-length E6

Since we had previously demonstrated in vitro interactions between HPV-18 E6*I and both fulllength HPV-18 E6 and E6-AP, we were interested in identifying which amino acid residues are required for each interaction. Figure 2 shows the E6* truncations which were made. These were expressed in bacteria as GST-fusion proteins, bound to glutathione resin, and assayed for their ability to bind in vitro translated HPV-18 E6 and E6-AP. The results obtained are shown in Figure 3. Truncations A to D are $\mathrm{N}$-terminal fragments of $\mathrm{E} 6 * \mathrm{I}$ of increasing length and neither HPV-18 E6 nor E6-AP showed any significant binding to fragments A, B or C. In contrast, binding of both HPV-18 E6 and E6-AP to fragment D at levels similar to that seen with wild-type E6*I indicates that this fragment comprises all the residues essential for both of these interactions. Similarly, using the C-terminal deletion fragments $\mathrm{E}, \mathrm{F}$ and $\mathrm{G}$, the binding of both HPV-18 E6 and E6-AP to E6*I is lost by the time E6*I has been truncated to fragment G. Taken together, these results suggest that the region spanning residues $21-43$ contains amino acids essential for the interactions between E6*I and both the full-length HPV-18 E6 and the E6-AP proteins.

In an attempt to differentiate between those residues of E6*I required for E6-AP binding and those required for HPV-18 E6 binding, we made three deletion mutants within this stretch of the E6*I polypeptide (shown in Figure 2), expressed them as GST fusion proteins and assessed their ability to bind to E6-AP

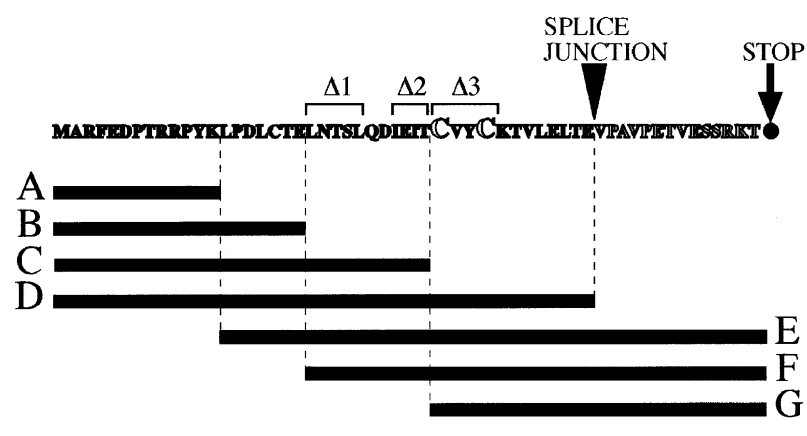

Figure 2 Truncation and deletion mutants of HPV-18 E6*I used in this study
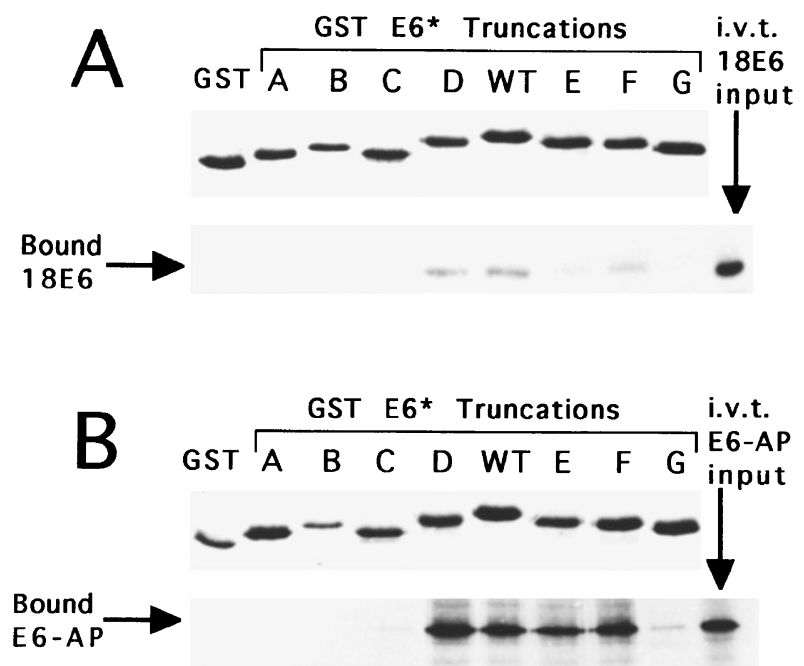

Figure 3 Binding of wild-type and truncation mutants of HPV18 E6*I expressed as GST-fusion proteins to: (a) in vitro transcribed/translated full-length HPV-18 E6, and (b) in vitro transcribed/translated E6-AP. After exposure gels were rehydrated, then stained to show GST-fusion protein inputs (upper part of each panel) 
and HPV-18 E6 in vitro. It can be seen from Figure 4a, that E6-AP binding to all three deletion mutants is reduced compared with the wild-type E6*I, with mutant $\Delta 2$ being the most strongly reduced. Figure $4 \mathrm{~b}$ shows the three deletion mutants binding to in vitro translated HPV-18 E6 and it is clear that mutant $\Delta 3$ binds with almost wild-type efficiency, $\Delta 1$ is somewhat reduced, and $\Delta 2$ is completely negative. These results show that all three deletions remove residues which contribute to the interaction between E6*I and E6-AP, whereas only the $\Delta 2$ mutation abolishes the interaction with full-length HPV-18 E6. Taken together, these
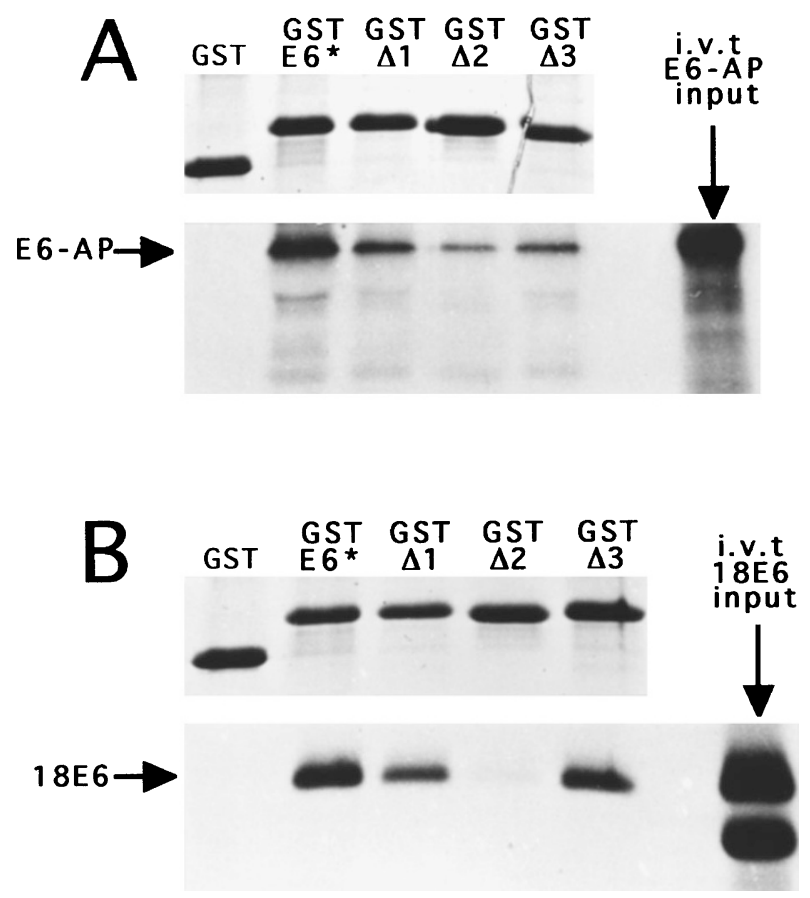

Figure 4 Binding of wild-type E6*I and deletion mutants $\Delta 1, \Delta 2$ and $\Delta 3$ expressed as GST-fusions, to: (a) in vitro transcribed/ translated E6-AP; (b) in vitro transcribed/translated full-length HPV-18 E6. After exposure gels were rehydrated, then stained to show GST-fusion protein inputs (upper part of each panel) results suggest that although the same general region of the E6*I polypeptide is involved in the interactions with both HPV-18 E6 and E6-AP, the exact residues essential for each interaction are not the same, with the $\Delta 2$ mutation in particular, allowing differentiation between the E6*I interactions with HPV-18 E6 and E6-AP.

$H P V-18 E^{*}$ mutant $\Delta 2$ is defective in its ability to inhibit the E6-directed degradation of p53 in vitro

We have previously shown that HPV-18 E6*I protein can inhibit the E6-directed degradation of p53 in vitro. Because HPV-18 E6*I mutant $\Delta 2$ is negative in its ability to bind to full-length HPV-18 E6, we were interested in examining its activity in this assay. We therefore incubated in vitro translated, radiolabelled p53 in the presence of in vitro translated full-length HPV-18 E6, which had been pre-incubated with an excess of either water primed lysate or in vitro translated wild-type E6*I, or mutants $\Delta 1, \Delta 2$ or $\Delta 3$. After $1 \mathrm{~h}$ the remaining p53 was determined by immunoprecipitation with an anti-p53 polyclonal antibody. The results obtained are shown in Figure 5 and demonstrate that while wild-type HPV-18 E6*I and mutants $\Delta 1$ and $\Delta 3$ are all able to inhibit the E6directed degradation of p53, mutant $\Delta 2$ is strongly reduced in this activity. These results indicate that the interaction between E6*I and the full-length HPV-18 E6 protein is necessary for the ability of E6*I to inhibit E6 directed degradation of p53 in vitro.

\section{$H P V-18$ E6*I mutant $\Delta 2$ is negative for the ability to reduce colony formation in CaSKi cells}

Having demonstrated that the E6*I $\Delta 2$ mutant was defective in its ability to inhibit E6 mediated degradation of $\mathrm{p} 53$, we were next interested in ascertaining its activity in vivo. We had previously shown that overexpression of HPV-18 E6*I protein has a growth suppressive effect in CaSKi cells, but not in cells lacking p53 and/or oncogenic E6 protein (Pim et al., 1997). It has been shown previously that p53 is not

\section{$2 \mu l$ of i.v.t. inputs}

WT E6*E6*E6*
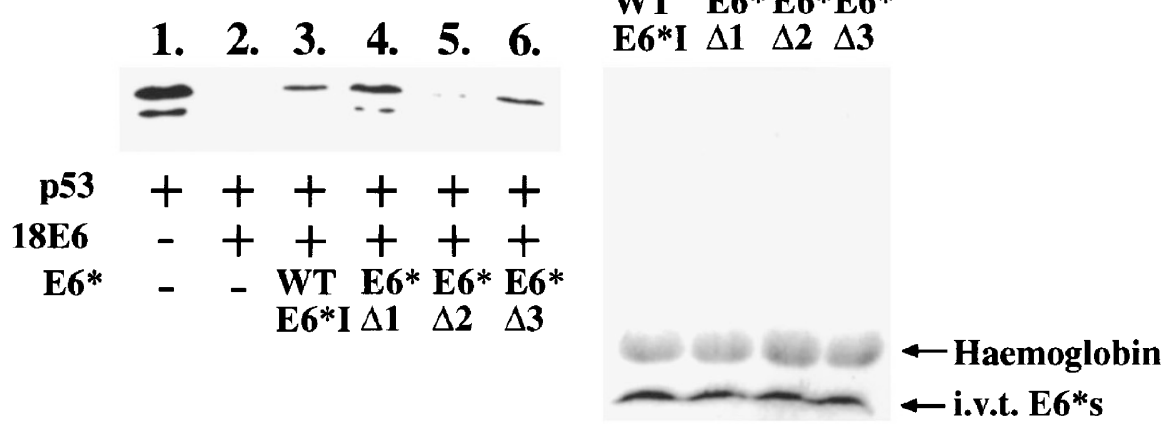

Figure 5 HPV-18 E6*I mutant $\Delta 2$ is reduced in its ability to inhibit the E6-directed degradation of p53 in vitro. Track 1: $4 \mu$ l of in vitro translated, radiolabelled p53 incubated with $23 \mu \mathrm{l}$ of water primed lysate. Track 2: $4 \mu 1$ of in vitro translated, radiolabelled p53 incubated with $3 \mu \mathrm{l}$ of in vitro translated HPV-18 E6 and $20 \mu \mathrm{l}$ of water primed lysate. Tracks $3-6: 4 \mu \mathrm{l}$ of in vitro translated, radiolabelled p53 incubated with $3 \mu \mathrm{l}$ of in vitro translated HPV-18E6 which had been pre-incubated with $20 \mu \mathrm{l}$ of in vitro translated wild-type (WT) E6*I or mutants $\Delta 1, \Delta 2$ or $\Delta 3$ as indicated. Residual p53 was visualized by immunoprecipitation and polyacrylamide gel electrophoresis 
readily activated in $\mathrm{HeLa}$ cells but that it can be activated in CaSKi cells (Butz et al., 1995; Pim et al., 1997; Mantovani and Banks, 1999). In addition, since the mutant E6*I proteins bound HPV-16 E6 in a similar manner to HPV-18 E6 (data not shown), we proceeded to investigate the antiproliferative capacities of the three mutants in a CaSKi cell colony-forming assay. CaSKi cells were transfected with constructs expressing wild-type E6*I and mutants $\Delta 1, \Delta 2$ and $\Delta 3$, then placed under G418 selection for 2 weeks before fixing, staining, and counting colonies. The results from four such experiments are shown in Figure 6 and show that, as previously reported, overexpression of E6*I in CaSKi cells has a marked antiproliferative effect. Mutants $\Delta 1$ and $\Delta 3$, which are both reduced for in vitro binding to E6-AP and HPV-18 E6, are also reduced in their ability to limit the proliferation of CaSKi cells whereas mutant $\Delta 2$ is completely negative in this assay. Since the $\Delta 2$ mutant is also negative for in vitro binding to HPV-18 E6 protein, yet retains binding to E6-AP, we conclude that binding to fulllength HPV-18 E6 is absolutely required for the antiproliferative properties of E6*I protein.

\section{The antiproliferative effect of HPV-18 E6*I in CaSKi cells is due to apoptosis}

We have previously demonstrated that E6*I expression leads to the transcriptional upregulation of p53 target genes in cells derived from cervical tumours (Pim et al., 1997). Since the induction of p53 leads to either cellcycle arrest (El-Deiry et al., 1993) or to the initiation of

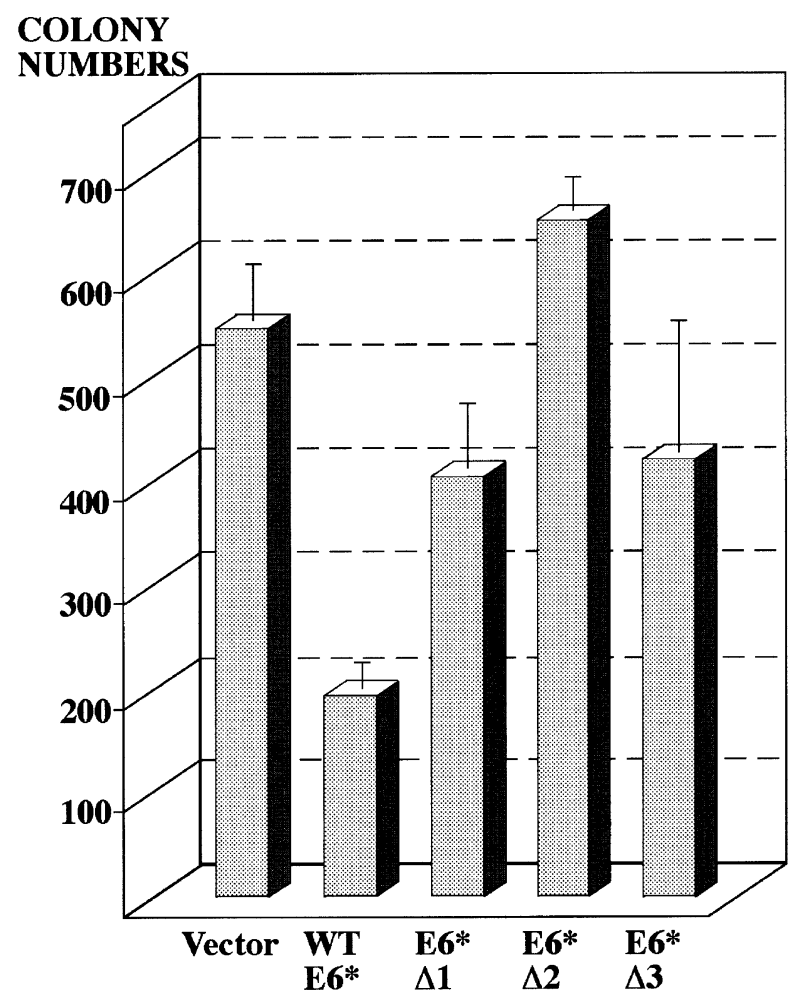

Figure 6 Numbers of G418-resistant colonies after transfection with pCDNA3 constructs expressing wild-type HPV-18 E6*I, E6*I $\Delta 1$, E6*I $\Delta 2$ and E6*I $\Delta 3$. The histogram represents the mean results of four separate experiments; standard deviations are shown apoptosis (Clarke et al., 1993; Lowe et al., 1993), we were interested in whether either of these pathways was responsible for the antiproliferative effects of E6*I. To address this question we transfected CaSKi and p53null Saos-2 cells with empty vector and constructs expressing wild-type HPV-18 E6*I and mutant E6*I $\Delta 2$. After $24 \mathrm{~h}$, cells were harvested, Annexin V stained and counted by FACS analysis. The results are shown in Figure 7. It can be seen that expression of neither wild-type E6*I, nor mutant $\Delta 2$ affects the Annexinbinding peak in Saos-2 cells relative to the control transfected cells. In contrast, in CaSKi cells, transfection with wild-type E6*I results in a large increase in the proportion of cells staining with Annexin V, whereas only a slight increase in the proportion of Annexin V staining cells is obtained following transfection with mutant $\Delta 2$. These results demonstrate that the antiproliferative effects of E6*I are due at least in part to the induction of apoptosis in a p53 dependent manner, and that this activity most likely occurs through complex formation with full-length HPV-16 E6 and inhibition of E6 mediated degradation of $\mathrm{p} 53$.

\section{Discussion}

We previously demonstrated that HPV-18 E6*I could inhibit E6 mediated degradation of p53 and suppress the growth of HPV transformed cells. To determine whether this activity was mediated by interaction with either full-length HPV E6, E6-AP or a combination of both, a detailed mutational analysis of the HPV-18 E6*I protein has been performed. This has localized the binding region for both E6-AP and HPV-18 E6 on E6*I to within a stretch of 20 amino acids. Within this region we made a set of three deletion mutants, $\Delta 1, \Delta 2$ and $\Delta 3$, and again assessed binding to HPV-18 E6 and E6-AP. The results from these studies demonstrate that

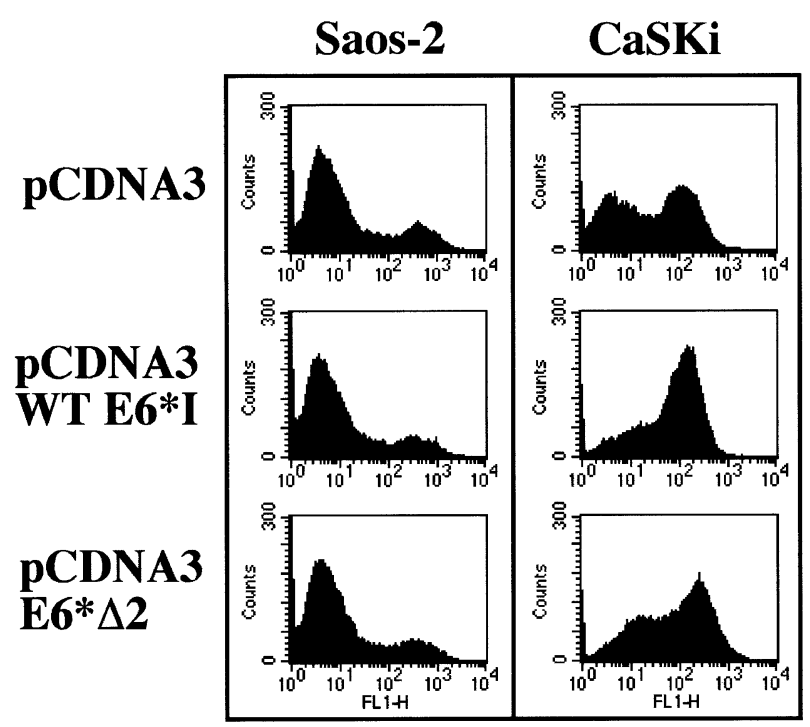

Figure 7 Overexpression of HPV-18 E6*I leads to apoptosis in CaSKi cells. CaSKi cells were electroporated with pCDNA3 and pCDNA3 constructs expressing wild-type HPV-18 E6*I and E6*I $\Delta 2$. After $24 \mathrm{~h}$ cells were harvested and analysed for Annexin Vbinding 
while interactions with both E6-AP and full-length E6 require residues lying within the same general region of the E6*I protein, the precise residues involved in each interaction are different. Thus, all three E6*I deletion mutants are reduced in their binding to E6-AP. In contrast, mutants $\Delta 1$ and $\Delta 3$ both bind strongly to HPV-18 E6 whereas mutant $\Delta 2$ is negative. We then analysed the ability of the three deletion mutants to inhibit the E6-directed degradation of $\mathrm{p} 53$ in vitro and found that both the wild-type E6*I and mutants $\Delta 1$ and $\Delta 3$ could inhibit the E6-directed degradation of p53, whereas mutant $\Delta 2$ had little effect. In order to assess the biological activity of the mutants, we carried out a series of colony forming assays in CaSKi cells. Interestingly, the results show that mutants $\Delta 1$ and $\Delta 3$ suppress the growth of CaSKi cells at reduced levels compared to that of wild-type E6*I, whereas mutant $\Delta 2$ was completely defective in this assay. The lack of effect of E6 ${ }^{*}$ mutant $\Delta 2$ on CaSKi cell proliferation correlates most closely with its reduced ability to inhibit the E6-directed degradation of $\mathrm{p} 53$ in vitro, and with its failure to bind HPV-18 E6. However, although binding to full-length E6 correlates most closely with inhibition of p53 degradation, the antiproliferative effects of E6*I would also appear to require the E6AP interaction. Thus, mutants $\Delta 1$ and $\Delta 3$ are both close to wild-type with respect to E6 binding, yet both are reduced in the ability to inhibit cell proliferation and bind E6-AP, albeit not to the same degree as the $\Delta 2$ mutation.

Having identified the mechanism by which HPV-18 E6*I exerts its antiproliferative effect, we were then interested in determining the form this effect might take when E6*I is overexpressed in CaSKi cells. This is a particularly relevant question as p53 has been shown to mediate both growth arrest and apoptotic pathways according to both the strength and nature of the genotoxic stimulus (Giaccia and Kastan, 1996 for review). In order to address this point, we compared the effect of expressing wild-type HPV-18 E6*I and mutant $\Delta 2$ in both p53-null Saos-2 cells and HPV-16containing CaSKi cells, and staining for Annexin V binding as a measure of apoptosis. In agreement with our previous studies, which showed that HPV-18 E6*I overexpression had no observable effect on the proliferation of Saos-2 cells, the proportion of Annexin V-binding cells was unaltered following E6*I transfection compared with control transfected cells. However, overexpression of E6*I in CaSKi cells leads to a marked increase in the proportion of cells binding Annexin V compared with the control-transfected cells, consistent with E6*I protecting p53 from full-length E6-directed degradation. In contrast, the E6*I mutant $\Delta 2$, which fails to bind full-length E6 and is unable to inhibit the proliferation of CaSKi cells, was strongly reduced in its ability to activate apoptosis. These results demonstrate that the E6*I inhibition of E6directed degradation of p53 is largely dependent upon the binding between E6*I and the full-length E6 protein and that, at least in CaSKi cells, the antiproliferative effects of HPV-18 E6*I overexpression are due in part to activation of apoptosis. At present we do not know whether a significant percentage of the cells also enter into growth arrest, although it has been shown previously that $\mathrm{p} 21$ protein is not induced by p53 in CaSKi cells (Butz et al., 1998), suggesting that G1 arrest is unlikely to play a major role in this activity of E6*I in these cells.

Whilst the antiproliferative effects of E6*I overexpression, due to the induction of apoptotis, represent a valid biological assay for its ability to inhibit E6 induced degradation of p53, an obvious caveat to these studies is whether such levels of E6*I are attained during viral infection. Nonetheless, since the region of HPV-18 E6*I which is required for its interaction with full-length E6 protein has now been identified, future studies are aimed at using synthetic peptides to block the E6-p53 interaction and at examining the use of such peptides to inhibit the growth of HPV-derived cervical tumour cells in vivo. The outcome of these studies will determine whether E6*I represents a valid candidate for use in gene therapy of HPV associated tumours.

\section{Materials and methods}

Cell lines

CaSKi and Saos-2 cells were grown and maintained in DMEM supplemented with $10 \%$ foetal calf serum.

\section{Plasmid constructs and mutagenesis}

The construction of the open reading frame for HPV-18 E6*I by PCR has been described previously (Pim et al., 1997). The cloning of this into vector pCDNA3 for in vivo expression, and into pSP64 for in vitro translation has also been previously described, as has the $95 \mathrm{kDa}$ E6-AP clone and the full-length HPV-18 E6 clone used for in vitro translation (Pim et al., 1997). The truncation and deletion mutants of HPV-18 E6*I used in this study were made by PCR, using the original wild-type E6*I clone (Pim et al., 1997), then subsequently cloned into bacterial expression vector pGEX2T, and verified by dideoxy DNA sequencing.

\section{In vitro binding assays}

Wild-type E6*I, and all the truncation and deletion mutants were expressed as GST-fusion proteins and purified as previously described (Pim et al., 1997). HPV-18 E6 and E6AP were in vitro transcribed and translated using the TNT reticulocyte lysate coupled transcription/translation system (Promega), incubated with the GST-fusion proteins bound to glutathione resin for $2 \mathrm{~h}$ at room temperature in a minimal volume of buffer containing $300 \mathrm{~mm} \mathrm{NaCl}, 50 \mathrm{~mm}$ Tris $\mathrm{pH}$ $7.5,1 \%$ Nonidet-P40 and $0.25 \%$ gelatin. After extensive washing in the same buffer, bound proteins were eluted by boiling and visualized by polyacrylamide gel electrophoresis.

\section{Inhibition of p53 degradation in vitro}

Wild-type p53, HPV-18 E6, wild-type HPV-18 E6*I and E6* mutants $\Delta 1, \Delta 2$ and $\Delta 3$ were in vitro transcribed and translated using the TNT rabbit reticulocyte lysate coupled transcription/translation system (Promega) in vitro degradation assays for $\mathrm{p} 53$ were done as previously described (Pim et al., 1997). Briefly, $4 \mu \mathrm{l}$ of in vitro translated, radiolabelled $\mathrm{p} 53$ was incubated for $1 \mathrm{~h}$ at $30^{\circ} \mathrm{C}$ with $3 \mu \mathrm{l}$ of in vitro translated HPV-18 E6 in the presence of $20 \mu$ of water primed lysate or $20 \mu \mathrm{l}$ of in vitro translated $\mathrm{E}^{*}$ or mutants $\Delta 1, \Delta 2$ or $\Delta 3$. After incubation, $1 \mu \mathrm{l}$ of anti-p53 antibody $\mathrm{C} 4$ was added for $1 \mathrm{~h}$ on ice, then immunoprecipitates bound to protein $\mathrm{A}$ sepharose (Pharmacia), washed, boiled, and the immunoprecipitated p53 visualized by polyacrylamide gel electrophoresis and autoradiography. 
CaSKi cells were plated at a density of $1 \times 10^{5}$ cells per $100 \mathrm{~mm}$ Petri dish, and transfected by Calcium Phosphatemediated transfer (Matlashewski et al., 1987), with pCDNA3, pCDNA3 E6*, and pCDNA3 E6* $\Delta 1, \Delta 2$ and $\Delta 3$. Cells were placed under selection with G418 at $200 \mu \mathrm{g} \mathrm{ml}^{-1}$ for 2 weeks before fixing with $10 \%$ formalin, staining with Giemsa, and counting colonies.

\section{Transfection of Saos-2 and CaSKi cells by electroporation}

Three confluent $150 \mathrm{~mm}$ Petri dishes each of CaSKi and Saos-2 cells were trypsinized, gently centrifuged and resuspended in $750 \mu \mathrm{l}$ each of DMEM containing 10\% BES (50 mM, pH 7.2). To $250 \mu \mathrm{l}$ of cell suspension in a Biorad gene-pulser cuvette was added $20 \mu \mathrm{g}$ of expression plasmid plus $10 \mu \mathrm{g}$ of herring sperm DNA as carrier, and the suspension was electroporated at $180 \mathrm{mV}$ in a Biorad gene pulser. The suspensions were chilled on ice for $10 \mathrm{~min}$ before washing once in DMEM, and then plating out on $100 \mathrm{~mm}$ Petri dishes. Cells were washed once in DMEM after $6 \mathrm{~h}$ to remove any cells damaged by electroporation. Transfection efficiencies were monitored in Saos- 2 cells by co-transfecting a lacZ expressing plasmid and staining for blue cells. This could not be performed in CaSKi cells due to the high degree of apoptosis induced by the wild-type E6*I construct.

\section{References}

Böhm S, Wilczynski S, Pfister H and Iftner T. (1993). Int. J. Cancer, 55, $791-798$.

Butz K, Shahabeddin L, Geisen C, Spitkovsky D, Ullmann A and Hoppe-Seyler F. (1995). Oncogene, 10, 927-936.

Butz K, Geisen C, Ullmann A, Zentgraf H and Hoppe-Seyler F. (1998). Oncogene, 17, $781-787$.

Cheng S, Schmidt-Grimminger D-C, Murant T, Broker T and Chow L. (1995). Genes Dev., 9, 2335-2349.

Clarke A, Purdie C, Harrison D, Morris R, Bird C, Hooper $\mathrm{M}$ and Wyllie A. (1993). Nature, 362, 849-852.

Doorbar J, Parton A, Hartley K, Banks L, Crook T, Stanley $\mathrm{M}$ and Crawford L. (1990). Virology, 178, 254-262.

Dyson N, Howley P, Munger K and Harlow E. (1989). Science, 243, 934-937.

El-Deiry W, Tokino T, Velculescu V, Levy D, Parsons R, Trent J, Lin D, Mercer W, Kinzler K and Vogelstein B. (1993). Cell, 75, $817-825$.

Giaccia A and Kastan M. (1998). Genes Dev., 12, 2973 2983.

Grassmann K, Rapp B, Maschek H, Petry K and Iftner T. (1996). J. Virol., 70, $2339-2349$.

Huibregtse J, Scheffner M and Howley P. (1991). EMBO J., 10, 4129-4135.

Lowe S, Ruley E, Jacks T and Houseman D. (1993). Cell, 74, $847-849$.

Mantovani F and Banks L. (1999). Oncogene, 18, $3309-$ 3315.

\section{Annexin V-binding assay for apoptosis}

Twenty-four hours after electroporation, the supernatants were removed from the dishes and retained. The cells were washed, and removed from the dishes with trypsin/EDTA, then pooled with their respective supernatants. The cell suspensions were spun gently, the pellets resuspended in $4 \mathrm{ml}$ of PBS and transferred to small reaction tubes. After a second spin, pellets were resuspended in $200 \mu \mathrm{l}$ of binding buffer containing $5 \mu \mathrm{l}$ of Annexin V-FITC reagent (Boehringer). Cells were incubated $10-15 \mathrm{~min}$ in the dark, a further $400 \mathrm{ml}$ of binding buffer added and then the cells were analysed for Annexin V-binding on a Fluorescence Activated Cell Sorter (Becton Dickinson).

\section{Acknowledgements}

We are most grateful to Miranda Thomas for comments on the manuscript. This work was supported in part by a research grant from the Associazione per la Ricerca sul Cancro.
Matlashewski G, Schneider J, Banks L, Jones N, Murray A and Crawford L. (1987). EMBO J., 6, 1741-1746.

Pim D, Massimi P and Banks L. (1997). Oncogene, 15, $257-$ 264.

Scheffner M, Werness B, Huibregtse J, Levine A and Howley P. (1990). Cell, 63, 1129-1136.

Scheffner M, Huibregtse J, Vierstra R and Howley P. (1993). Cell, 75, 495-505.

Schneider-Gädicke A and Schwarz E. (1986). EMBO J., 5, $2285-2292$.

Schwarz E, Freese U, Gissman L, Mayer W, Roggenbuck B, Stremlau A and zur Hausen H. (1985). Nature, 314, 111 114

Sherman L and Alloul N. (1992). Virology, 191, 953-959.

Smotkin D and Wettstein F. (1986). Proc. Nat. Acad. Sci. USA, 83, 4680-4684.

Smotkin D, Prokoph H and Wettstein F. (1989). J. Virol., 63, $1441-1447$.

Stacey S, Jordan D, Snijders P, Mackett M, Walboomers J and Arrand J. (1995). J. Virol., 69, $7023-7031$.

Werness B, Levine A and Howley P. (1990). Science, 248, $76-79$.

zur Hausen H and Schneider A. (1987). In: Salzman NP and Howley PM. (eds). The Papillomaviruses. Plenum Publishing Corp.: New York, pp. 245-263. 